\title{
TAMANHO DE GOTAS DE PONTAS DE PULVERIZAÇÃO EM DIFERENTES CONDIÇÕES OPERACIONAIS POR MEIO DA TÉCNICA DE DIFRAÇÃO DO RAIO LASER
}

\section{MARIANA R. BUENO ${ }^{1}$, JOÃO P. A. R. DA CUNHA ${ }^{2}$, RODRIGO A. A. ROMAN ${ }^{3}$}

RESUMO: O presente trabalho objetivou avaliar o espectro de gotas de pontas de pulverização hidráulicas, submetidas a diferentes pressões e composições de calda, por meio de um analisador de partículas a laser. Em delineamento inteiramente casualizado, avaliaram-se duas pontas de jato plano duplo com indução de ar (AD-IA/D 11002 e AD-IA/D 11004) e duas pontas de jato cônico vazio (MAG - 2 e MAG - 4), em esquema fatorial 3 x 2: três pressões de pulverização (207, 276 e $345 \mathrm{kPa}$ para as pontas de jato plano duplo e 414; 483 e $552 \mathrm{kPa}$ para as pontas de jato cônico); e duas composições de calda (água e água mais o adjuvante fosfatidilcoline + ácido propiônico). A adição do adjuvante reduziu o diâmetro da mediana volumétrica das gotas pulverizadas pelas pontas AD-IA/D 11002 e 11004; porém, teve efeito inverso com a ponta MAG - 4 e não o alterou com a ponta MAG - 2. Em condições climáticas adversas, não se recomenda o uso de pontas de jato cônico vazio, mesmo com a adição do adjuvante testado, em virtude do alto risco potencial de deriva.

PALAVRAS-CHAVE: espectro de gotas, tecnologia de aplicação, adjuvante.

\section{DROPLET SIZE OF SPRAY NOZZLES IN DIFFERENT OPERATIONAL CONDITIONS USING LASER DIFRACTION TECHNIQUE}

\begin{abstract}
The present study aimed to evaluate the droplet spectrum of hydraulic nozzles, under different pressures and spray liquid compositions, using a laser particle size analyzer. In a completely randomized design, two air induction twin flat-fan nozzles (AD-IA/D 11002 and ADIA/D11004) and two hollow-cone nozzles (MAG - 2 and MAG - 4) were evaluated, in factorial design $3 \times 2$ : tree spray pressures $(207,276$ and $345 \mathrm{kPa}$ for twin flat-fan nozzles, and 414, 483 and $552 \mathrm{kPa}$ for cone nozzles); and two spray liquid compositions (water and water plus phosphatydilcoline + propionic acid adjuvant). The addition of adjuvant reduced the volume median diameter for the AD-IA/D 11002 and 11004 nozzles; however it had an opposite effect with the MAG - 4 nozzles and not changed with the MAG - 2 nozzles. In adverse weather conditions, it is not recommended the use of hollow cone spray nozzle, even with the addition of adjuvant tested because of the high risk potential of drift.
\end{abstract}

KEYWORDS: droplet size, spray technology, surfactant.

\section{INTRODUÇÃO}

Uma correta aplicação de produtos fitossanitários somente é possível quando se dispõe de pontas de pulverização que proporcionem distribuição transversal homogênea e espectro de gotas adequado (NUYTTENS et al., 2007; CUNHA \& SILVA; 2010). Contudo, nem sempre o tamanho das gotas produzidas segue uma distribuição uniforme, podendo ser produzidas gotas finas, médias e grossas por uma única ponta.

Pontas de jato cônico vazio e de jato plano produzem espectro de gotas bastante diferentes, sendo que a maior diferença é encontrada na porcentagem de gotas pequenas (MATTHEWS, 2000;

\footnotetext{
${ }^{1}$ Eng a Agrônoma, Doutoranda, Instituto de Ciências Agrárias, Universidade Federal de Uberlândia, Uberlândia-MG, marianarb_agro@yahoo.com.br.

${ }^{2}$ Eng $^{\circ}$ Agrícola, Prof. Doutor, Instituto de Ciências Agrárias, Universidade Federal de Uberlândia, Uberlândia-MG, jpcunha@iciag.ufu.br.

${ }^{3}$ Eng $^{\circ}$ Agrônomo, Mestre, Departamento de Fitossanidade, FCAV/UNESP, Jaboticabal-SP, alandiaroman @ gmail.com. Recebido pelo Conselho Editorial em: 30-9-2010

Aprovado pelo Conselho Editorial em: 5-4-2013
} 
STAINIERA et al., 2006). Alguns pesquisadores consideram que gotas menores que $100 \mu \mathrm{m}$ são facilmente carregadas pelo vento, sofrendo mais intensamente a ação dos fenômenos climáticos (MURPHY et al., 2000; CUNHA et al., 2004).

As pontas de jato cônico vazio produzem, em geral, gotas finas, que apresentam trajetórias tangenciais, que facilitam a penetração das gotas no dossel das plantas (ZHU et al., 2004). No entanto, quando há presença de gotas finas em uma aplicação, há risco de contaminação ambiental e humana em função da deriva e, por isso, têm-se buscado alternativas que minimizem tais problemas (STAINIERA et al., 2006; NUYTTENS et al., 2007).

Uma das alternativas para reduzir esses riscos é a utilização de pontas que produzem gotas de maior tamanho, como as pontas de jato plano duplo com indução de ar. A princípio, gotas extremamente grossas são indicadas para aplicação de herbicidas em pré e pós-emergência e dessecação em condições climáticas desfavoráveis (VIANA et al., 2007), não devendo ser empregadas quando se requer elevada cobertura do alvo. Contudo, o processo de contato dessas gotas com inclusão de ar no alvo pode levar ao incremento da área de contato e, assim, aumentar seu potencial de uso com fungicidas e inseticidas.

Autores como CUNHA et al. (2008) e JULIATTI et al. (2010), avaliando diferentes pontas de pulverização no controle de doenças na cultura da soja e do milho, respectivamente, verificaram que o uso de pontas com indução de ar tem efeito similar no controle de doenças, na produtividade da cultura e na massa de 1.000 grãos, quando comparado ao uso de pontas que produzem gotas finas ou médias. Também KNEWITZ et al. (2002), em uma série de experimentos conduzidos durante três anos, concluíram que o uso de pontas com indução de ar não diminuiu a eficácia biológica em aplicações visando ao controle das principais pragas e doenças da macieira.

Durante uma aplicação em campo, outro fator que também influencia o espectro de gotas é a adição de adjuvantes à calda de pulverização. Com a utilização desses produtos, busca-se melhorar a penetração, a aderência e o espalhamento da calda (PENNER, 2000; RYCKAERT et al., 2007) e reduzir a tensão superficial das gotas, permitindo maior contato entre as gotas e a superfície da folha pulverizada (WAGNER et al., 2003; VAN ZYL et al., 2010).

Grande parte dos problemas advindos da utilização de adjuvantes de calda advém do fato de que muitos dos produtos disponíveis no mercado não têm o devido respaldo científico. Além disso, o efeito dos adjuvantes pode ser distinto em função do tipo de ponta empregado. Esses produtos alteram as características físico-químicas da calda, principalmente viscosidade e tensão superficial (CUNHA \& ALVES et al., 2009), modificando, por consequência, o espectro de gotas geradas (FERREIRA et al., 2009; VIANA et al., 2010).

Desse modo, o presente trabalho teve como objetivo avaliar o espectro de gotas de pontas de pulverização hidráulica de jato plano duplo com indução de ar e de jato cônico vazio, submetidas a diferentes pressões e composições de calda.

\section{MATERIAL E MÉTODOS}

O presente trabalho foi realizado no Laboratório de Análise de Tamanho de Partículas (LAPAR), do Departamento de Fitossanidade da Faculdade de Ciências Agrárias e Veterinárias Câmpus de Jaboticabal - SP, da Universidade Estadual Paulista.

$\mathrm{O}$ experimento foi conduzido em delineamento inteiramente casualizado. Foram avaliados quatro modelos de pontas: duas pontas de jato plano duplo com indução de ar (AD-IA/D 11002 e AD-IA/D 11004) e duas pontas de jato cônico vazio (MAG - 2 e MAG - 4), todas da empresa Magnojet. Foram escolhidos quatro exemplares novos de cada modelo de ponta, cada qual correspondente a uma repetição. Para cada tipo de ponta, empregou-se um esquema fatorial $3 \times 2$, sendo três pressões de pulverização $(207 ; 276$ e $345 \mathrm{kPa}$ para as pontas de jato plano e 414; 483 e $552 \mathrm{kPa}$ para as pontas de jato cônico vazio) e duas composições de calda (água e água mais o adjuvante fosfatidilcoline + ácido propiônico $\left(712,88 \mathrm{~g} \mathrm{~L}^{-1}\right)$, na dose recomendada de $0,5 \mathrm{~L}_{100 \mathrm{~L}^{-1}}$. 
A adição desse adjuvante (Nome comercial LI 700), de acordo com o fabricante, tem como objetivo a redução da tensão superficial das gotas de pulverização, promovendo maior contato das gotas com o alvo, além de, também, atuar como agente acidificador de calda e redutor de deriva (AGROFIT, 2013).

A análise do espectro de gotas foi realizada de forma direta, utilizando um analisador de partículas em tempo real Mastersizer $S^{\circledR}$ (Malvern Instruments Ltd.). O diâmetro das gotas é proporcional ao ângulo do desvio sofrido pelo raio laser dessa forma, quanto menor a partícula, maior é o ângulo de desvio que o raio de luz sofre (ETHERIDGE et al., 1999).

O equipamento dispõe de unidade óptica constituída por uma lente focal. Para a medição do espectro de gotas das pontas AD-IA/D, utilizou-se da lente focal de $1.000 \mathrm{~mm}$, com capacidade de mensurar gotas com diâmetro de 4 a $3.000 \mu \mathrm{m}$. O bico de pulverização foi instalado a $40 \mathrm{~cm}$ de distância do feixe de laser, e foi movimentado $55^{\circ}$ para a direita e para a esquerda, com o intuito de que todo o jato atravessasse transversalmente o feixe durante a leitura. Para as pontas MAG, utilizou-se de uma lente focal de $300 \mathrm{~mm}$, capaz de analisar gotas na faixa de 0,5 a $900 \mu \mathrm{m}$. Para manter a pressão hidráulica constante, utilizou-se de ar comprimido, advindo de um compressor (Bravo CSL15BR, Schulz, Joinville, Brasil) controlado com regulador de pressão de precisão, operado manualmente, munido de manômetro analógico (Edmansfort, São Paulo, Brasil), de forma semelhante à empregada por FERREIRA et al. (2007). O conjunto de calibração estava instalado no circuito de pressurização antes do tanque de calda.

A decodificação dos dados, segundo o algoritmo elaborado para a caracterização do diâmetro de partículas por difração de raios laser, foi realizada pelo programa computacional Mastersizer $S$ versão 2.19, Malvern Instruments Ltd. Os valores relacionados ao espectro de tamanho de partículas foram processados e tabulados diretamente pelo programa, sendo apresentados de forma tabelada.

Foram processadas duas leituras para cada ponta e realizada a média dos resultados das análises. Avaliaram-se os seguintes parâmetros: $\mathrm{Dv}_{0,1}$ - diâmetro de gota tal que $10 \%$ do volume do líquido pulverizado é constituído de gotas de tamanho menor que esse valor; $\mathrm{Dv}_{0,5}$ - diâmetro de gota tal que $50 \%$ do volume do líquido pulverizado é constituído de gotas de tamanho menor que esse valor, também conhecido como diâmetro da mediana volumétrica (DMV); $\mathrm{Dv}_{0,9}$ - diâmetro de gota tal que $90 \%$ do volume do líquido pulverizado é constituído de gotas de tamanho menor que esse valor; AR - amplitude relativa, e $\mathrm{Dv}<100 \mu \mathrm{m}$ - percentagem do volume de gotas pulverizado com diâmetro inferior a $100 \mu \mathrm{m}$. A característica $\mathrm{Dv}_{0,1}$ é importante em avaliações de espectro de gotas, pois indica que, quanto maior for este valor, menor será o risco potencial de deriva da aplicação (CAMARA et al., 2008).

Os dados de espectro de gotas foram primeiramente submetidos aos testes de normalidade de Shapiro Wilk e homogeneidade das variâncias de Levene. Em seguida, procedeu-se à análise de variância (ANOVA) a 0,05 de probabilidade e, constatada diferença significativa, as médias das características em estudo foram comparadas pelo teste de Tukey, também a 0,05 de probabilidade.

\section{RESULTADOS E DISCUSSÃO}

As condições ambientais durante a realização dos ensaios foram: temperatura do ar variando entre 26 e $28^{\circ} \mathrm{C}$, umidade relativa do ar entre 70 e $75 \%$, e ausência de ventos e de luminosidade externa, uma vez que as análises foram realizadas com ausência de luz.

$\mathrm{Na}$ Tabela 1, encontram-se os diâmetros médios dos volumes acumulados de $10 \%$ e $50 \%$ para os quatro modelos de pontas estudados. Para a variável $\mathrm{Dv}_{0,1}$, apenas na ponta AD-IA/D 11002, a interação entre os fatores pressão e adjuvante foi significativa, indicando a dependência entre os dois fatores; para as demais pontas, a interação não foi significativa, o que implica a não dependência entre os mesmos. Já para a variável $\mathrm{Dv}_{0,5}$, as duas pontas de jato plano duplo com indução de ar (AD-IA/D 11002 e 11004) apresentaram interação significativa entre os fatores, enquanto para as pontas de jato cônico vazio (MAG - 2 e 4) não houve interação significativa. 
Percebe-se que, para o $\mathrm{Dv}_{0,1}$ e o $\mathrm{Dv}_{0,5}$, o tamanho de gotas foi reduzido à medida que se aumentaram as pressões de 207 a $345 \mathrm{kPa}$, para as pontas de jato plano duplo com indução de ar, e de 414 a $552 \mathrm{kPa}$, para as pontas de jato cônico vazio. A única exceção foi a não alteração do $\mathrm{Dv}_{0,1}$ das gotas pulverizadas pela ponta AD-IA/D 11002 nas três pressões em estudo, quando se adicionou adjuvante à calda.

TABELA 1. Distribuição volumétrica por classe de tamanho $\left(\mathrm{Dv}_{0,1}\right.$ e $\left.\mathrm{Dv}_{0,5}\right)$ das gotas pulverizadas por pontas de jato plano duplo com indução de ar e jato cônico vazio, com e sem a adição de adjuvante à calda. Volumetric droplet size distribution $\left(\mathbf{D v}_{\mathbf{0 , 1}}\right.$ and $\left.\mathbf{D v _ { 0 , 5 }}\right)$ for air induction twin flat-fan and hollow-cone nozzles, with and without adjuvant addition in spray liquid.

\begin{tabular}{|c|c|c|c|c|c|c|c|}
\hline \multirow{3}{*}{$\begin{array}{l}\text { Pontas de } \\
\text { Aplicação }\end{array}$} & \multirow{3}{*}{$\begin{array}{c}\text { Pressão } \\
\text { (kPa) }\end{array}$} & \multicolumn{3}{|c|}{$\mathbf{D v}_{0,1}(\mu \mathrm{m})$} & \multicolumn{3}{|c|}{$\mathbf{D v}_{0,5}(\mu \mathrm{m})$} \\
\hline & & & Adjuvante & & & Adjuvante & \\
\hline & & Sem & Com & Média & Sem & Com & Média \\
\hline \multirow{6}{*}{$\begin{array}{l}\text { AD-IA/D } \\
11002\end{array}$} & 207 & $171,68 \mathrm{Cb}$ & $96,16 \mathrm{Aa}$ & 133,92 & $543,52 \mathrm{Cb}$ & $309,82 \mathrm{Ba}$ & 426,67 \\
\hline & 276 & $129,25 \mathrm{Bb}$ & $82,55 \mathrm{Aa}$ & 105,90 & $420,81 \mathrm{Bb}$ & $232,93 \mathrm{ABa}$ & 326,87 \\
\hline & 345 & $98,99 \mathrm{Ab}$ & $76,89 \mathrm{Aa}$ & 87,94 & $290,39 \mathrm{Ab}$ & 177,32 Aa & 233,86 \\
\hline & Média & 133,31 & 85,20 & & 418,24 & 240,03 & \\
\hline & & \multicolumn{3}{|c|}{$\mathrm{CV}=12,85 \%$} & \multicolumn{3}{|c|}{$\mathrm{CV}=13,28 \%$} \\
\hline & & $\mathrm{F}_{\mathrm{P}}=21,794 * *$ & \multicolumn{2}{|c|}{$\mathrm{F}_{\mathrm{A}}=70,435^{* *} \mathrm{~F}_{\mathrm{PxA}}=7,254 * *$} & \multicolumn{3}{|c|}{$\begin{array}{c}\mathrm{F}_{\mathrm{P}}=38,915 * * \quad \mathrm{~F}_{\mathrm{A}}=99,699 * * \\
\mathrm{~F}_{\mathrm{PxA}}=3,880 *\end{array}$} \\
\hline \multirow{6}{*}{$\begin{array}{l}\text { AD-IA/D } \\
11004\end{array}$} & 207 & 126,26 & 112,61 & $119,44 \mathrm{~B}$ & $436,95 \mathrm{Bb}$ & $322,01 \mathrm{Ba}$ & 379,48 \\
\hline & 276 & 108,07 & 94,64 & $101,35 \mathrm{~A}$ & $329,98 \mathrm{Ab}$ & $280,62 \mathrm{ABa}$ & 305,30 \\
\hline & 345 & 104,15 & 86,23 & $95,19 \mathrm{~A}$ & $273,71 \mathrm{Aa}$ & $246,02 \mathrm{Aa}$ & 259,86 \\
\hline & Média & $112,83 \mathrm{~b}$ & $97,83 \mathrm{a}$ & & 346,88 & 282,88 & \\
\hline & & \multicolumn{3}{|c|}{$\mathrm{CV}=6,83 \%$} & \multicolumn{3}{|c|}{$\mathrm{CV}=10,32 \%$} \\
\hline & & $\mathrm{F}_{\mathrm{P}}=24,576 * *$ & $\mathrm{~F}_{\mathrm{A}}=26,115^{* *}$ & $\mathrm{~F}_{\mathrm{PxA}}=0,248^{\mathrm{ns}}$ & \multicolumn{3}{|c|}{$\begin{array}{c}\mathrm{F}_{\mathrm{P}}=27,622 * * \quad \mathrm{~F}_{\mathrm{A}}=23,273 * * \\
\mathrm{~F}_{\mathrm{PxA}}=3,909 *\end{array}$} \\
\hline \multirow{6}{*}{ MAG - 2} & 414 & 44,76 & 41,91 & $43,34 \mathrm{~B}$ & 113,91 & 128,74 & $121,32 \mathrm{~B}$ \\
\hline & 483 & 42,91 & 37,91 & $40,41 \mathrm{AB}$ & 103,86 & 108,26 & $106,06 \mathrm{AB}$ \\
\hline & 552 & 39,63 & 30,66 & $35,15 \mathrm{~A}$ & 97,17 & 85,43 & $91,30 \mathrm{~A}$ \\
\hline & Média & $42,43 \mathrm{~b}$ & $36,83 \mathrm{a}$ & & 104,98 a & $107,47 \mathrm{a}$ & \\
\hline & & \multicolumn{3}{|c|}{$\mathrm{CV}=10,73 \%$} & \multicolumn{3}{|c|}{$\mathrm{CV}=11,69 \%$} \\
\hline & & $\mathrm{F}_{\mathrm{P}}=7,621 * *$ & $\mathrm{~F}_{\mathrm{A}}=10,428 * *$ & $\mathrm{~F}_{\mathrm{PxA}}=1,066^{\mathrm{ns}}$ & $\mathrm{F}_{\mathrm{P}}=11,689 * *$ & $\mathrm{~F}_{\mathrm{A}}=0,242^{\mathrm{ns}}$ & $\mathrm{F}_{\mathrm{PxA}}=2,322^{\mathrm{ns}}$ \\
\hline \multirow{6}{*}{ MAG - 4} & 414 & 48,03 & 42,13 & $45,08 \mathrm{~B}$ & 141,42 & 160,69 & $151,06 \mathrm{~B}$ \\
\hline & 483 & 45,29 & 39,38 & $42,33 \mathrm{~B}$ & 131,71 & 144,68 & $138,20 \mathrm{~A}$ \\
\hline & 552 & 39,60 & 37,78 & $38,69 \mathrm{~A}$ & 122,91 & 130,30 & $126,61 \mathrm{~A}$ \\
\hline & Média & $44,31 \mathrm{~b}$ & $39,76 \mathrm{a}$ & & $132,02 \mathrm{a}$ & $145,23 \mathrm{~b}$ & \\
\hline & & \multicolumn{3}{|c|}{$\mathrm{CV}=6,33 \%$} & \multicolumn{3}{|c|}{$\mathrm{CV}=6,51 \%$} \\
\hline & & $\mathrm{F}_{\mathrm{P}}=11,607 * *$ & $\mathrm{~F}_{\mathrm{A}}=17,482 * *$ & $\mathrm{~F}_{\mathrm{PxA}}=1,578^{\mathrm{ns}}$ & $\mathrm{F}_{\mathrm{P}}=14,691 * *$ & $\mathrm{~F}_{\mathrm{A}}=12,857 * *$ & $\mathrm{~F}_{\mathrm{PxA}}=0,866^{\mathrm{ns}}$ \\
\hline
\end{tabular}

$\mathrm{CV}$ : coeficiente de variação; $\mathrm{F}_{\mathrm{P}}$ : valor do $\mathrm{F}$ calculado para o fator pressão; $\mathrm{F}_{\mathrm{A}}$ : valor do $\mathrm{F}$ calculado para o fator adjuvante; $\mathrm{F}_{\mathrm{PxA}}$ : valor do F calculado para a interação entre os fatores pressão e adjuvante. Médias seguidas por letras distintas maiúsculas, nas colunas, e minúsculas, nas linhas, diferem entre si, pelo teste de Tukey, a 0,05 de probabilidade. $* *$ significativo a 0,01 ; $*$ significativo a 0,$05 ;{ }^{\text {ns }}$ não significativo.

CUNHA et al. (2007), estudando pontas de jato plano (API e ADI) e jato cônico vazio (ATR), verificaram que estas apresentaram comportamento de espectro de gotas diferenciado. Nas pontas de jato plano, o diâmetro das gotas diminuiu com o incremento da pressão, corroborando os 
resultados deste trabalho. Porém, observaram que o aumento da pressão nas pontas de jato cônico vazio não influenciou sobre o diâmetro das gotas nas pressões estudadas. Os autores justificam que a magnitude de variação de tamanho de gotas em função da pressão nas pontas de jato cônico vazio é menor do que nas pontas de jato plano.

FERREIRA et al. (2009) e VIANA et al. (2010), também estudando o espectro de gotas de pontas hidráulicas (TT, TTI e AI; e TTI, AI e AIV, respectivamente), em diferentes pressões e composições de calda, observaram que o tamanho de gotas foi reduzido com o aumento da pressão (até $400 \mathrm{kPa}$ ).

Nota-se também uma redução significativa do diâmetro médio das gotas do volume acumulado de $10 \%$, quando se adicionou o adjuvante fosfatidilcoline + ácido propiônico à calda de pulverização, para todas as pontas e pressões estudadas. A adição do adjuvante, em geral, reduziu o $\mathrm{Dv}_{0,5}$ das gotas pulverizadas pelas pontas de jato plano duplo com indução de ar AD-IA/D $11002 \mathrm{e}$ 11004, mas teve efeito inverso com a ponta de jato cônico vazio MAG - 4 e não o alterou com a utilização da ponta de jato cônico vazio MAG - 2 .

FERREIRA et al. (2009), ao avaliarem o diâmetro das gotas produzidas por pontas de pulverização hidráulica com caldas de diferentes adjuvantes, verificaram que a adição do adjuvante fosfatidilcoline + ácido propiônico à calda reduziu o tamanho das gotas produzidas por pontas de jato plano defletor com indução de ar - TTI, mas não alterou o diâmetro das pontas de jato plano com indução de ar - AI e jato plano de grande ângulo - TF-2. Já IOST (2008), avaliando o efeito de adjuvantes com potencial antideriva (nonilfenoletoxilado+óxido de etileno, fosfatidilcoline+ácido propiônico, nonil fenol etoxilado, lecitina e polímero glicolsilano) no tamanho de gotas, notou pouco efeito no diâmetro da mediana volumétrica, nas doses recomendadas pelos fabricantes.

Percebe-se grande diferença quanto ao desempenho das pontas de jato plano duplo com indução de ar e de jato cônico vazio quando há presença do adjuvante na calda. Para as pontas de indução de ar (com exceção do tratamento citado anteriormente), o adjuvante tem a capacidade de reduzir significamente o tamanho médio das gotas pulverizadas; porém, quando a aplicação é realizada com a ponta de jato cônico vazio $\mathrm{MAG}-4$, o adjuvante aumentou o $\mathrm{Dv}_{0,5}$ das gotas.

Possivelmente, essa diferenciação ocorreu devido à complexidade do processo de formação de gotas em pontas de indução de ar, ainda pouco conhecido e estudado. O sistema venturi presente nestas pontas, combinado ao pré-orificio, permite formação de gotas de maior tamanho com pequenas bolhas de ar em seu interior, tendo comportamento diferente de gotas sólidas (NUYTTENS et al., 2007). A maioria dos adjuvantes com função de espalhante tem em sua composição propriedades redutoras de tensão superficial. Essa, por sua vez, pode levar também à diminuição do tamanho das gotas; contudo, a magnitude deste processo não é muito grande e varia de acordo com a ponta empregada (BUTLER-ELLIS et al., 2001). Esse fato ajuda a explicar o comportamento apresentado pelas pontas estudadas.

O diâmetro médio do volume acumulado de $90 \%$ e a percentagem do volume pulverizado, composto por gotas pulverizadas de tamanho menor que $100 \mu \mathrm{m}$, são detalhados na Tabela 2. Para ambas as variáveis, não houve interação significativa entre pressão e adjuvante, o que indica que esses fatores são independentes um do outro.

Com relação ao $\mathrm{Dv}_{0,9}$, é perceptível a redução do diâmetro médio das gotas pulverizadas à medida que se aumentou a pressão de trabalho para as quatro pontas em estudo. Os diâmetros médios variaram de 554,60 a $879,48 \mu \mathrm{m}$ para gotas produzidas pelas pontas de jato plano duplo com indução de ar e de 206,76 a 318,37 $\mu \mathrm{m}$ para as pontas de jato cônico vazio.

Para as pontas de jato plano duplo, a adição do adjuvante à calda reduziu significamente o diâmetro médio das gotas do volume acumulado de $90 \%$, já que, como constatado anteriormente, esse adjuvante tem a capacidade de reduzir o diâmetro médio $\left(\mathrm{Dv}_{0,5}\right)$ das gotas produzidas por esse tipo de ponta (Tabela 1). Já para as pontas de jato cônico vazio, houve diferença de comportamento entre as duas pontas, seguindo o mesmo já observado anteriormente para a variável $\mathrm{Dv}_{0,5}$. Para a 
ponta MAG - 2, a utilização do adjuvante não interferiu no $\mathrm{Dv}_{0,9}$ das gotas, mas para a ponta MAG - 4, o mesmo adjuvante, quando presente na calda, aumentou o valor do $\mathrm{Dv}_{0,9}$.

TABELA 2. Distribuição volumétrica por classe de tamanho $\left(\mathrm{Dv}_{0,9}\right)$ e percentagem do volume pulverizado, composto por gotas com diâmetro inferior a $100 \mu \mathrm{m}$, por pontas de jato plano duplo, com indução de ar e jato cônico vazio, com e sem a adição de adjuvante à calda. Volumetric droplet size distribution $\left(\mathrm{Dv}_{0,9}\right)$ and percentage of spray volume in droplets smaller than $100 \mu \mathrm{m}$ diameter for air induction twin flat-fan and hollow-cone nozzles, with and without adjuvant addition in spray liquid.

\begin{tabular}{|c|c|c|c|c|c|c|c|}
\hline \multirow{3}{*}{$\begin{array}{l}\text { Pontas de } \\
\text { Aplicação }\end{array}$} & \multirow{3}{*}{$\begin{array}{c}\text { Pressão } \\
\text { (kPa) }\end{array}$} & \multicolumn{3}{|c|}{$\mathbf{D v}_{0,9}(\mu \mathrm{m})$} & \multicolumn{3}{|c|}{ Gotas $<100 \mu \mathrm{m}(\%)$} \\
\hline & & & Adjuvante & & & Adjuvante & \\
\hline & & Sem & Com & Média & Sem & Com & Média \\
\hline \multirow{6}{*}{$\begin{array}{c}\text { AD-IA/D } \\
11002\end{array}$} & 207 & 1017,84 & 739,13 & $879,48 \mathrm{C}$ & 3,67 & 11,06 & $7,36 \mathrm{~A}$ \\
\hline & 276 & 906,91 & 558,95 & $732,93 \mathrm{~B}$ & 5,80 & 14,65 & $10,23 \mathrm{AB}$ \\
\hline & 345 & 766,56 & 342,64 & $554,60 \mathrm{~A}$ & 10,52 & 21,45 & 15,98 B \\
\hline & Média & $897,10 \mathrm{~b}$ & $546,91 \mathrm{a}$ & & 6,66 a & $15,72 \mathrm{~b}$ & \\
\hline & & \multicolumn{3}{|c|}{$C V=15,16 \%$} & \multicolumn{3}{|c|}{$\mathrm{CV}=53,48 \%$} \\
\hline & & $\mathrm{F}_{\mathrm{P}}=17,574 * *$ & $\mathrm{~F}_{\mathrm{A}}=61,428 * *$ & $\mathrm{~F}_{\mathrm{PxA}}=0,881^{\mathrm{ns}}$ & $\mathrm{F}_{\mathrm{P}}=4,304^{*}$ & $\mathrm{~F}_{\mathrm{A}}=13,729 * *$ & $\mathrm{~F}_{\mathrm{PxA}}=0,177^{\mathrm{ns}}$ \\
\hline \multirow{6}{*}{$\begin{array}{c}\text { AD-IA/D } \\
11004\end{array}$} & 207 & 991,21 & 720,89 & $856,05 \mathrm{~B}$ & 5,84 & 7,98 & $6,91 \mathrm{~A}$ \\
\hline & 276 & 816,62 & 639,93 & $728,28 \mathrm{AB}$ & 8,32 & 11,06 & $9,69 \mathrm{~B}$ \\
\hline & 345 & 712,25 & 563,70 & $637,98 \mathrm{~A}$ & 9,07 & 13,57 & $11,32 \mathrm{~B}$ \\
\hline & Média & $840,03 \mathrm{~b}$ & $641,51 \mathrm{a}$ & & $7,74 \mathrm{a}$ & $10,87 \mathrm{~b}$ & \\
\hline & & \multicolumn{3}{|c|}{$\mathrm{CV}=16,30 \%$} & \multicolumn{3}{|c|}{$\mathrm{CV}=15,74 \%$} \\
\hline & & $\mathrm{F}_{\mathrm{P}}=6,589 * *$ & $\mathrm{~F}_{\mathrm{A}}=16,222 * *$ & $\mathrm{~F}_{\mathrm{PxA}}=0,558^{\mathrm{ns}}$ & $\mathrm{F}_{\mathrm{P}}=18,569 * *$ & $\mathrm{~F}_{\mathrm{A}}=27,293^{* *}$ & $\mathrm{~F}_{\mathrm{PxA}}=1,393^{\mathrm{ns}}$ \\
\hline \multirow{6}{*}{ MAG - 2} & 414 & 256,06 & 268,40 & $262,23 \mathrm{~B}$ & 43,07 & 37,70 & $40,39 \mathrm{~A}$ \\
\hline & 483 & 228,32 & 242,94 & $235,63 \mathrm{AB}$ & 47,92 & 46,33 & $47,13 \mathrm{AB}$ \\
\hline & 552 & 217,23 & 196,30 & 206,76 A & 51,62 & 60,97 & $56,30 \mathrm{~B}$ \\
\hline & Média & $233,87 \mathrm{a}$ & $235,88 \mathrm{a}$ & & $47,54 \mathrm{a}$ & $48,34 \mathrm{a}$ & \\
\hline & & \multicolumn{3}{|c|}{$\mathrm{CV}=11,87 \%$} & \multicolumn{3}{|c|}{$\mathrm{CV}=17,40 \%$} \\
\hline & & $\mathrm{F}_{\mathrm{P}}=7,925 * *$ & $\mathrm{~F}_{\mathrm{A}}=0,031^{\mathrm{ns}}$ & $\mathrm{F}_{\mathrm{PxA}}=1,020^{\mathrm{ns}}$ & $\mathrm{F}_{\mathrm{P}}=7,331 * *$ & $\mathrm{~F}_{\mathrm{A}}=0,055^{\mathrm{ns}}$ & $\mathrm{F}_{\mathrm{PxA}}=1,680^{\mathrm{ns}}$ \\
\hline \multirow{6}{*}{ MAG - 4} & 414 & 308,87 & 327,87 & $318,37 \mathrm{~B}$ & 33,33 & 30,10 & $31,71 \mathrm{~A}$ \\
\hline & 483 & 292,66 & 308,80 & $300,73 \mathrm{~A}$ & 36,63 & 34,28 & $35,46 \mathrm{~A}$ \\
\hline & 552 & 283,24 & 292,14 & $287,69 \mathrm{~A}$ & 40,37 & 38,35 & $39,36 \mathrm{~B}$ \\
\hline & Média & 294,93 a & $309,60 \mathrm{~b}$ & & $36,78 \mathrm{a}$ & $34,24 \mathrm{a}$ & \\
\hline & & \multicolumn{3}{|c|}{$\mathrm{CV}=3,99 \%$} & \multicolumn{3}{|c|}{$\mathrm{CV}=8,22 \%$} \\
\hline & & $\mathrm{F}_{\mathrm{P}}=13,065^{* *}$ & $\mathrm{~F}_{\mathrm{A}}=8,905 * *$ & $\mathrm{~F}_{\mathrm{PxA}}=0,373^{\mathrm{ns}}$ & $\mathrm{F}_{\mathrm{P}}=13,712 * *$ & $\mathrm{~F}_{\mathrm{A}}=4,515^{\mathrm{ns}}$ & $\mathrm{F}_{\mathrm{PxA}}=0,092^{\mathrm{ns}}$ \\
\hline
\end{tabular}

CV: coeficiente de variação; $F_{P}$ : valor do $F$ calculado para o fator pressão; $F_{A}$ : valor do $F$ calculado para o fator adjuvante; $F_{P x A}$ : valor do $\mathrm{F}$ calculado para a interação entre os fatores pressão e adjuvante. Médias seguidas por letras distintas maiúsculas, nas colunas, e minúsculas, nas linhas, diferem entre si, pelo teste de Tukey, a 0,05 de probabilidade. $* *$ significativo a 0,01 ; * significativo a 0,$05 ;{ }^{\text {ns }}$ não significativo.

CÂMARA et al. (2008), estudando o espectro de gotas de bicos hidráulicos de jato plano de faixa expandida, observaram maior valor do $\mathrm{D}_{\mathrm{V} 0,9}$ para a pressão de $200 \mathrm{kPa}$ com a adição de surfactantes com propriedade tensoativa, mas esse não diferiu, na pressão de $400 \mathrm{kPa}$, do tratamento com água. 
A interpretação dos dados de percentagem do volume de gotas com diâmetro inferior a 100 $\mu \mathrm{m}$ (Tabela 2) permite que se estime o potencial de deriva da aplicação. Assim, nota-se que as pontas de jato cônico vazio estão relativamente mais sujeitas à deriva que as pontas de indução de ar, independentemente da pressão de trabalho, e que, quanto maior essa pressão, maior a porcentagem de gotas aspergidas menor que $100 \mu \mathrm{m}$.

Quanto menor essa percentagem, menor o risco de deriva durante uma aplicação de produto fitossanitário, uma vez que gotas menores que $100 \mu \mathrm{m}$ são mais propensas à deriva, sofrendo mais intensamente a ação dos fenômenos climáticos (MURPHY et al., 2000; CUNHA et al., 2004).

Em geral, valores inferiores a $15 \%$ do volume pulverizado composto por gotas com diâmetro inferior a $100 \mu \mathrm{m}$ parecem ser mais adequados a uma aplicação segura (CUNHA et al., 2003). Portanto, o uso de pontas de jato cônico vazio deve ser feito com critério, evitando-se aplicações em condições ambientais adversas como: temperatura do ar maior que $30^{\circ} \mathrm{C}$, umidade relativa menor que $55 \%$ e vento com velocidade acima de $12 \mathrm{~km} \mathrm{~h}^{-1}$.

TABELA 3. Amplitude relativa das gotas pulverizadas por pontas de jato plano duplo com indução de ar e jato cônico vazio, com e sem a adição de adjuvante à calda. Relative width of droplets for air induction twin flat-fan and hollow-cone nozzles, with and without adjuvant addition in spray liquid.

\begin{tabular}{|c|c|c|c|c|}
\hline \multirow{3}{*}{$\begin{array}{l}\text { Pontas de } \\
\text { Aplicação }\end{array}$} & \multirow{3}{*}{ Pressão (kPa) } & \multicolumn{3}{|c|}{ Amplitude Relativa (AR) } \\
\hline & & & Adjuvante & \\
\hline & & Sem & Com & Média \\
\hline \multirow{6}{*}{ AD-IA/D } & 207 & $1,560 \mathrm{Aa}$ & $2,068 \mathrm{Bb}$ & 1,814 \\
\hline & 276 & $1,848 \mathrm{ABa}$ & $2,028 \mathrm{Ba}$ & 1,938 \\
\hline & 345 & 2,322 Bb & 1,484 Aa & 1,903 \\
\hline & Média & 1,910 & 1,860 & \\
\hline & & \multicolumn{3}{|c|}{$\mathrm{CV}=14,57 \%$} \\
\hline & & $F_{P}=0,436^{n s}$ & $\mathrm{~F}_{\mathrm{A}}=0,197^{\mathrm{ns}}$ & $\mathrm{F}_{\mathrm{PxA}}=13,057 * *$ \\
\hline \multirow{6}{*}{$\begin{array}{c}\text { AD-IA/D } \\
11004\end{array}$} & 207 & 1,991 & 1,865 & $1,921 \mathrm{~A}$ \\
\hline & 276 & 2,147 & 1,912 & $2,030 \mathrm{~A}$ \\
\hline & 345 & 2,211 & 2,045 & $2,130 \mathrm{~A}$ \\
\hline & Média & $2,117 \mathrm{a}$ & $1,941 \mathrm{a}$ & \\
\hline & & & $\mathrm{CV}=13,30 \%$ & \\
\hline & & $\mathrm{F}_{\mathrm{P}}=1,107^{\mathrm{ns}}$ & $\mathrm{F}_{\mathrm{A}}=2,536^{\mathrm{ns}}$ & $\mathrm{F}_{\mathrm{PXA}}=0,084^{\mathrm{ns}}$ \\
\hline \multirow{6}{*}{ MAG - 2} & 414 & 1,855 & 1,765 & $1,810 \mathrm{~A}$ \\
\hline & 483 & 1,784 & 1,912 & $1,848 \mathrm{~A}$ \\
\hline & 552 & 1,830 & 1,894 & $1,862 \mathrm{~A}$ \\
\hline & Média & $1,823 \mathrm{a}$ & $1,857 \mathrm{a}$ & \\
\hline & & & $\mathrm{CV}=8,85 \%$ & \\
\hline & & $\mathrm{F}_{\mathrm{P}}=0,218^{\mathrm{ns}}$ & $\mathrm{F}_{\mathrm{A}}=0,259^{\mathrm{ns}}$ & $\mathrm{F}_{\mathrm{PxA}}=0,952^{\mathrm{ns}}$ \\
\hline \multirow{6}{*}{ MAG - 4} & 414 & 1,827 & 1,779 & $1,803 \mathrm{~A}$ \\
\hline & 483 & 1,878 & 1,874 & $1,876 \mathrm{AB}$ \\
\hline & 552 & 1,984 & 1,952 & $1,968 \mathrm{~B}$ \\
\hline & Média & $1,897 \mathrm{a}$ & $1,869 \mathrm{a}$ & \\
\hline & & & $\mathrm{CV}=3,96 \%$ & \\
\hline & & $\mathrm{F}_{\mathrm{P}}=9,873 * *$ & $\mathrm{~F}_{\mathrm{A}}=0,853^{\mathrm{ns}}$ & $\mathrm{F}_{\mathrm{PXA}}=0,181^{\mathrm{ns}}$ \\
\hline
\end{tabular}

$\mathrm{CV}$ : coeficiente de variação; $\mathrm{F}_{\mathrm{P}}$ : valor do $\mathrm{F}$ calculado para o fator pressão; $\mathrm{F}_{\mathrm{A}}$ : valor do $\mathrm{F}$ calculado para o fator adjuvante; $\mathrm{F}_{\mathrm{PxA}}$ : valor do $\mathrm{F}$ calculado para a interação entre os fatores pressão e adjuvante. Médias seguidas por letras distintas maiúsculas, nas colunas, e minúsculas, nas linhas, diferem entre si, pelo teste de Tukey, a 0,05 de probabilidade. ** significativo a 0,01 ; * significativo a 0,$05 ;{ }^{\text {ns }}$ não significativo. 
Novamente, confirma-se o comportamento do adjuvante de reduzir o tamanho das gotas emitidas pelas duas pontas de jato plano duplo com indução de ar, já que, com a adição do mesmo, houve maior porcentagem de gotas pulverizadas menores que $100 \mu \mathrm{m}$. $\mathrm{O}$ mesmo não aconteceu com as duas pontas de jato cônico vazio, para as quais não houve diferença quanto à utilização de adjuvante. A redução de tamanho de gotas é desejada principalmente quando o objetivo da aplicação é proporcionar boa cobertura ao alvo, uma vez que gotas menores proporcionam maior cobertura. Entretanto, em condições climáticas adversas, não se recomenda a utilização de pontas de jato cônico vazio, mesmo com a adição do adjuvante testado, em virtude do elevado risco de deriva.

Encontram-se, na Tabela 3, os valores médios de amplitude relativa das pontas estudadas. Apenas para a ponta AD-IA/D 11002, a interação entre os dois fatores avaliados foi significativa, demonstrando dependência entre os mesmos.

Os valores médios de amplitude relativa tiveram um comportamento diferente em função da calda de aplicação. Para a calda sem o adjuvante, a maior pressão, seguida da pressão intermediária (345 e $276 \mathrm{kPa}$ ) obtiveram as maiores amplitudes relativas. Em contrapartida, quando a calda estava com adjuvante, a menor pressão, seguida da pressão intermediária (207 e $276 \mathrm{kPa}$ ) proporcionaram os maiores valores de amplitude relativa.

Já para a ponta MAG - 4, os valores de amplitude relativa diferiram apenas na variável pressão, e quanto ao uso do adjuvante na calda, não houve diferença. Os menores valores de amplitude foram observados quando as pontas foram submetidas às pressões de 414 e $483 \mathrm{kPa}$.

Para as pontas AD-IA/D 11004 e MAG - 2, não houve diferença significativa para nenhum dos fatores envolvidos na análise. De forma geral, é possível afirmar que, nas condições avaliadas, o adjuvante não propiciou aumento da uniformidade das gotas geradas. FERREIRA et al. (2009) também não encontraram alteração nos valores de amplitude relativa para as pontas TT, TTI e AI com adição do adjuvante fosfatidilcoline + ácido propiônico à calda de pulverização.

\section{CONCLUSÕES}

Nas condições operacionais deste ensaio e na maioria dos tratamentos, a adição do adjuvante fosfatidilcoline + ácido propiônico à calda reduziu o diâmetro da mediana volumétrica das gotas pulverizadas pelas pontas de jato plano duplo com indução de ar AD-IA/D 11002 e 11004, mas teve efeito inverso com a ponta de jato cônico vazio MAG - 4 e não o alterou com a ponta de jato cônico vazio MAG - 2.

A uniformidade das gotas geradas pelas pontas de jato cônico vazio não foi influenciada pela adição do adjuvante à calda. Com relação às pontas de jato plano duplo com indução de ar, os resultados variaram em função da pressão empregada e da vazão nominal da ponta.

\section{AGRADECIMENTOS}

Ao CNPq, à Fapemig e à Capes, pelo suporte financeiro que permitiu o desenvolvimento deste trabalho de pesquisa.

\section{REFERÊNCIAS}

AGROFIT. Consulta de produtos formulados. Disponível em: <http://agrofit.agricultura.gov.br/agrofit_cons/principal_agrofit_cons>. Acesso em: $21 \mathrm{fev} .2013$.

BUTLER ELLIS, M.C.; TUCK, C.R.; MILLER, P.C.H. How surface tension of surfactant solutions influences the characteristics of sprays produced by hydraulic nozzles used for pesticide application. Colloids and Surfaces A: Physicochemical and Engineering Aspects, London, v. 190, $\mathrm{n}$. 3, p. 267-276, 2001. 
CAMARA, F.T.; SANTOS, J.L.; SILVA, E.A.; FERREIRA, M.C. Distribuição volumétrica e espectro de gotas de bicos hidráulicos de jato plano de faixa expandida XR 11003. Engenharia Agrícola, Jaboticabal, v. 28, n. 4, p. 740-749, 2008.

CUNHA, J.P.A.R.; SILVA, R.A.M. Uniformidade de distribuição volumétrica de pontas de pulverização em função da pressão de trabalho e altura da barra. Bioscience Journal, Uberlândia, v. 26, n. 1, p. 52-58, 2010.

CUNHA, J.P.A.R.; ALVES, G.S. Características fisico-químicas de soluções aquosas com adjuvantes de uso agrícola. Interciência, Caracas, v. 34, n. 9, p. 655-659, 2009.

CUNHA, J.P.A.R.; MOURA, E. A. C.; SILVA JÚNIOR, J. L.; ZAGO, F. A.; JULIATTI. F. C. Efeito de pontas de pulverização no controle químico da ferrugem da soja. Engenharia Agrícola, Jaboticabal, v. 28, n. 2, p. 283-291, 2008.

CUNHA, J.P.A.R.; TEIXEIRA, M.M.; FERNADES, H.C. Avaliação do espectro de gotas de pontas de pulverização hidráulicas utilizando a técnica da difração do raio laser. Engenharia Agrícola, Jaboticabal, v. 27, p. 10-15, 2007. Número especial.

CUNHA, J.P.A.R.; TEIXEIRA, M.M.; COURY, J.R.; FERREIRA, L.R. Avaliação de estratégias para redução da deriva de agrotóxicos em pulverizações hidráulicas. Planta Daninha, Viçosa, v. 21, n. 2, p. 325-332, 2003.

CUNHA, J.P.A.R.; TEIXEIRA, M.M.; VIEIRA, R.F.; FERNANDES, H.C.; COURY, J.R. Espectro de gotas de bicos de pulverização hidráulicos de jato plano e de jato cônico vazio. Pesquisa Agropecuária Brasileira, Brasília, v. 39, n. 10, p. 977-985, 2004.

ETHERIDGE, R. E.; WOMAC, A. R.; MUELLER, T. C. Characterization of the spray droplet spectra and patterns of four venturi-type drift reduction nozzles. Weed Technology, Lawrence, v. 13, n. 4, p. 765-70, 1999.

FERREIRA, M. C.; COSTA, G. M.; SILVA, A. R.; TAGLIARI, S. R. A. Fatores qualitativos da ponta de energia hidráulica ADGA 110015 para pulverização agrícola. Engenharia Agrícola, Jaboticabal, v. 27, n. 2, p. 471-478, 2007.

FERREIRA, M.C.; FERNANDES, A.P.; ROMÁN, R.A.A. Potencial de deriva das gotas grossas produzidas por pontas de pulverização de energia hidráulica, com caldas de diferentes adjuvantes. In: CONGRESSO BRASILEIRO DE ENGENHARIA AGRÍCOLA, 38., 2009, Juazeiro, Petrolina. Anais... Juazeiro, Petrolina: Associação Brasileira de Engenharia Agrícola, 2009. 1 CD-ROM.

IOST, C.A.R. Efeito de adjuvantes nas propriedades físico-químicas da água e na redução de deriva em pulverizações sobre diferentes espécies de plantas daninhas. 2008. 62 f. Dissertação (Mestrado em Agronomia/Proteção de Plantas) - Universidade Estadual Paulista, Faculdade de Ciências Agronômicas, Botucatu, 2008.

JULIATTI, F. C.; NASCIMENTO, C.; REZENDE, A. A. Avaliação de diferentes pontas e volumes de pulverização na aplicação de fungicida na cultura do milho. Summa Phytopathologica, Botucatu, v. 36, n. 3, p. 216-221, 2010.

KNEWITZ, H.; WEISSER, P.; KOCH, H. Drift-reducing spray application in orchards and biological efficacy of pesticides. Aspects of Applied Biology, Wellesbourne, n. 66, p. 231-236, 2002.

MATTHEWS, G. A. Pesticide application methods. 2nd ed. Oxford: Blackwell, 2000. 405p.

MURPHY, S.D.; MILLER, P.C.H.; PARKIN, C.S. The effect of boom section and nozzle configuration on the risk of spray drift. Journal of Agricultural Engineering Research, London, v. 75 , n. 2, p. $127-37,2000$. 
NUYTTENS, D.; BAETENS, K.; DE SCHAMPHELEIRE, M.; SONCK, B. Effect of nozzle type, size and pressure on spray droplet characteristics. Biosystems Engineering, Silsoe, v. 97, n. 3, p. 333-345, 2007.

PENNER, D. Activator adjuvants. Weed Technology, Washington, v. 14, n. 4, p. 785-791, 2000.

RYCKAERT, B.; SPANOGHE, P.; HAESAERT, G.; HEREMANS, B.; ISEBAERT, S.; STEURBAUT, W. Quantitative determination of the influence of adjuvants on foliar fungicide residues. Crop Protection, London, v. 26, n. 10, p. 1.589-1.594, 2007.

STAINIERA, C.; DESTAINA, M.F.; SCHIFFERSB, B.; LEBEAUA, F. Droplet size spectra and drift effect of two phenmedipham formulations and four adjuvants mixtures. Crop Protection, London, v. 25, n. 12, p. 1.238-1.243, 2006.

VAN ZYL, S.A.; BRINK, J; CALITZ, F.J.; COERTZE, S.; FOURIE, P.H. The use of adjuvants to improve spray deposition and Botrytis cinerea control on Chardonnay grapevine leaves. Crop Protection, London, v. 29, n.1, p. 58-67, 2010.

VIANA, R.G.; FERREIRA, L.R.; FERREIRA, M.C.; TEIXEIRA, M.M.; ROSELL, J.R.; TUFFI SANTOS, L.D.; MACHADO, A.F.L. Distribuição volumétrica e espectro de gotas de pontas de pulverização de baixa deriva. Planta Daninha, Viçosa-MG, v. 28, n. 2, p. 439-446, 2010.

VIANA, R.G.; FERREIRA, L.R.; TEIXEIRA, M.M.; CECON, P.R.; FREITAS, F.C.L.; QUIRINO, A.L.S.; SANTOS, M.V. Características técnicas de pontas de pulverização LA-1JC e SR-1. Planta Daninha, Viçosa, v. 25, n. 1, p. 211-218, 2007.

WAGNER, P.; FÜRSTNER, R.; BARTHLOTT. W.; NEINHUIS, C.J. Quantitative assessment to the structural basis of water repellency in natural and technical surfaces. Journal of Experimental Botany, Oxford, v. 54, n. 385, p. 1.295-1.303, 2003.

ZHU, H.; DORNER, J.W.; ROWLAND, D.L.; DERKSEN, R.C.; OZKAN, H.E. Spray penetration into peanut canopies with hydraulic nozzle tips. Biosystems Engineering, Silsoe, v. 87, n. 3, p. 275283, 2004. 